\title{
THE WADA TEST
}

\section{Contributions to standardization of the stimulus for language and memory assessment}

\author{
Maria Joana Mäder1, Bellkiss Wilma Romano², \\ Luciano De Paola 3 , Carlos Eduardo Soares Silvado ${ }^{4}$
}

\begin{abstract}
The Wada Test (WT) is part of the presurgical evaluation for refractory epilepsy. The WT is not standardized and the protocols differ in important ways, including stimulus type of material presented for memory testing, timing of presentations and methods of assessment. The aim of this study was to contribute to establish parameters for a WT to Brazilian population investigating the performance of 100 normal subjects, without medication. Two parallel models were used based on Montreal Procedure adapted from Gail Risse's (MEG-MN,EUA) protocol. The proportions of correct responses of normal subjects submitted to two parallel WT models were investigated and the two models were compared. The results showed that the two models are similar but significant differences among the stimulus type were observed. The results suggest that the stimulus type may influence the results of the WT and should be considered when constructing models and comparing different protocols.
\end{abstract}

KEY WORDS: neuropsychology, amobarbital test, epilepsy, memory,Wada test.

\section{Teste de Wada: contribuição para normatização dos estímulos para avaliação de linguagem e memória}

\begin{abstract}
RESUMO - O Teste de Wada (TW) é parte integrante da avaliação pré-cirúrgica para epilepsias de difícil controle. O TW não é padronizado e os protocolos diferem em vários aspectos, incluindo tipos de estímulos para avaliação de memória, tempo de apresentação e metodologia de avaliação. O objetivo deste estudo foi contribuir no estabelecimento de parâmetros para utilização TW para a população brasileira, investigando o desempenho de 100 controles normais, sem medicação. Dois modelos (Modelos A e B) foram utilizados, baseados no Procedimento de Montreal e adaptadas do protocolo de Gail Risse (MEG-MN,EUA). Foram observadas as proporções de acerto dos sujeitos normais para os modelos de TW, em seguida, os dois modelos foram comparados entre si. Os resultados demonstraram que os dois modelos são similares mas, observaram-se significativas diferenças entre os tipos de estímulos. Os resultados sugerem que tipos de estímulos podem influenciar os resultados do TW e esses dados devem ser considerados na construção de modelos e comparações entre diferentes protocolos.
\end{abstract}

PALAVRAS-CHAVE: neuropsicologia, teste do amobarbital, epilepsia, memória, teste de Wada.

Most epilepsy patients' seizures may be controlled by proper medication. Those whose seizures cannot be controlled by the available drugs are surgery candidates; however, not all patients with such characteristics may truly benefit from surgery $^{1}$. For a correct indication for surgery in refractory epilepsies, especially of the temporal lobe, a thorough investigation is required, involving a multidisciplinary team. Several exams are necessary: clinical analysis; imaging (MRI); neurophysiologic (video-electroencephalography), and neuropsychological, including the Wada Test (WT). Videoelectroencephalography is performed to study the association between the epileptic seizure's clinical manifestation and the electroencephalographic record, and is very valuable to locate seizure onset

Pesquisa realizada no Programa de Cirurgia de Epilepsia do Hospital de Clínicas da Universidade Federal do Paraná, Curitiba PR Brasil (UFPR): ${ }^{1}$ Psicóloga UFPR; ${ }^{2}$ Diretora do Serviço de Psicologia do Instituto do Coração do Hospital das Clínicas da Faculdade de Medicina da Universidade de São Paulo SP Brasil (INCOR USP); ${ }^{3}$ Chefe do Serviço de Eletroencefalografia UFPR; ${ }^{4}$ Coordenador do Programa de Cirurgia de Epilepsia UFPR.

Received 18 September 2003, received in final form 13 February 2004. Accepted 18 March 2004.

Dra. Maria Joana Mäder - Hospital de Clínicas UFPR - Rua General Carneiro 181 - 80060-900 Curitiba PR - Brasil. E-mail: mjmader@onda.com.br 
areas. MRI enables the assessment of hippocampal structures, indicating if there is mesial temporal sclerosis. Neuropsychological evaluation aims at investigating cognitive functions, especially material-specific memory, evidencing signs of function location and lateralization ${ }^{2,3}$. The WT basically consists of evaluating language and memory, while each cerebral hemisphere is temporarily inactivated by an injection of amobarbital into the internal carotid artery, so that both brain hemispheres can be evaluated independently. By anesthetizing one of the hemispheres it is possible to evaluate the other's function, providing data on language and memory lateralization. While the left hemisphere is anesthetized, the right hemisphere is in charge of memorizing information and vice-versa ${ }^{4-6}$.

Technically, the exam is performed by placing a catheter into the internal carotid arteries through a puncture in the femoral artery. The catheter is positioned in the internal carotid artery and amobarbital is injected. The hemisphere ipsilateral to the side of proposed surgery is anesthetized first. Since amobarbital is a fast acting barbiturate, the time the patient remains anesthetized (and consequently the testing time) is limited to a few minutes. Anesthetic efficacy can be determined by the time it takes for full return of motor function, and the presence of memory and language can only be interpreted while the opposite hemisphere is paralyzed, which is clinically measured by the motor strength of the contralateral upper limb?

Although most multidisciplinary teams who specialize in epilepsy surgery use the WT for evaluation of both memory and language, the way results are interpreted, as weel as the amount of amobarbital used tend to vary considerably. There are significant variations among the criteria used to consider positive or negative results. Some protocols base the final result on the correct answer rate (correctly memorized stimuli), while others base the results on the asymmetry between the correct answer rate obtained in both hemispheres ${ }^{8,9}$. A more detailed review on the WT's history and different protocols may be found in a previous publication ${ }^{10}$. Considering those aspects, a study was carried out to investigate normal subjects' responses to WT models: Model A and Model B. The structure of the exam was based on the exam created by Risse and collaborators ${ }^{11}$, but using different words and pictures. The words were selected among nouns, adjectives, adverbs and verbs, terms that are common in the vocabulary but difficult to see as an image, that is, difficult to associate to an image or object. The pictures in Risse's protocol are black and white outline designs but we chose to use colored pictures of objects, animals or plants.

The overall objective of this study was to provide a parameter of two WT models to be used for the Brazilian population. The first specific objective was to check the correct answer rate among a population that had had elementary and secondary education, for language and memory stimuli, with both WT models (Model A and Model B). The second specific objective was to compare subjects' performance in both models, checking the consistency of results to evaluate asymmetry of performance between the two hemispheres. Lastly, the third specific objective was to compare correct answer rates among different types of stimuli.

\section{METHOD}

The WT protocol used in our study was the Montreal Procedure with an additional interview stage; part of the stimuli were adapted from Risse's Protocol ${ }^{11}$, from the Minnesota Epilepsy Group (Saint Paul, MN, USA).

One hundred subjects with elementary and secondary education (either complete or incomplete), of both genders, ages ranging from 18 and 50, were recruited among the employees and volunteers of HC UFPR (University Hospital of the Federal University of Paraná). We chose as eligible subjects those who had no neurological complaints or symptoms, and whose results were equal or above the cut-off point proposed by Bertolucci et al. ${ }^{12}$ for the Mini Mental Status Examination (MMSE). All subjects agreed with Informed Consent before starting the test sessions.

Although the subjects were off medication, the procedure followed the same stimuli presentation order in the real situation of the WT. The time interval between stimuli presentation and patient recall, as well as the time between application of the two models were filled with short distractive tasks.

Model A consisted in presenting two series of stimuli, for 5 five seconds each, in the following order: 2 colored objects of original size and shape; 2 words to read and repeat; 2 abstract designs and 2 pictures of common objects. In between the two series, simple tasks of language stimulation were introduced, e.g. asking subjects to name the days of the week and identify geometrical shapes.

After the second series of stimuli was presented, the Trail Making Test ${ }^{13,14}$ was introduced as the distractive task. Subsequently, subjects were asked to simply recall the stimuli presented in both series. After recalling the stimuli, and even if all elements had been recalled, patients were asked to recognize the stimuli: eight objects were presented one by one, interposed between the 4 stimuli-objects, to be recognized through "yes" or "no" 
answers. Soon after, cards with 4 words each were presented and subjects were asked to recognize them giving multiple choice answers. Each abstract drawing was also presented printed on a card with three similar ones for recognition.

The pictures were to be recognized in two stages. First they were presented in written form together with three other words, and then each picture was presented together with three other different ones of the same category. For example: the picture of an airplane was presented as initial stimulus; in the recognition phase, a card was presented containing four words: train - airplane - can - ring and, immediately after that a card containing four different airplane pictures was presented for a multiple choice answer.

As soon as Model A was concluded, a new distractive task was applied, the Five Points ${ }^{13,14}$ completing a 30 minute interval since the beginning of the test. Right after that, Model B was applied with the Stroop Test ${ }^{13,14}$ as distractive task. All subjects responded to both models, following the stimuli presentation and distractive task order.

The language tests used both in Models A and B are the same and very simple. Basically, they include spontaneous speech exercises (object naming and word reading), automatic speech (saying the days of the week and repeating words), and verbal comprehension (pointing to geometric forms following verbal orders).

For memory assessment, verbal and visual stimuli recalled through free recall and recognition were grouped. The types of stimuli were assessed separately as well. The results were separated by stimulus type: Word Recognition; Abstract Drawing Recognition; Picture Verbal Recognition (recognizing the names of the pictures presented), and Picture Recognition.

\section{RESULTS}

Analysis of results for Models $A$ and $B$ - First we examined the right answer rate in recall and recognition for Models A and B. Then, we compared the results obtained in the two models, investigating the similarity between them. At last, we compared the rate of right answers in evocation and recognition of the different types of stimuli to check which types yielded the highest rate of correct answers.

Demographic data - The study included 56 women and 44 men with elementary and secondary education. Ninety-two subjects were employees at the Hospital's various departments, and 8 volunteers from the Hospital's Volunteer Service were included, as well. Table 1 shows the sample's distribution by gender and education level. Considering the responses could be different depending on the subject's education level, we chose to analyze the WT results by grouping men and women who
Table 1. Sample's distribution by gender and education.

\begin{tabular}{llll}
\hline & \multicolumn{2}{c}{ Gender } & \\
\cline { 2 - 3 } Schooling & Women & Men & Total \\
\hline Education group & & & \\
$\quad$ Elementary & 31 & 22 & 53 \\
Secondary & 25 & 22 & 47 \\
Total & 56 & 44 & 100 \\
\hline
\end{tabular}

had either completed elementary education or not in the Elementary Education Group. In the Secondary Education Group we included all men and women regardless if they had completed secondary education or not.

Rate of right answers for Models $A$ and $B$ - Results obtained in the language tests showed minimal incidence of errors, as we had expected, since the tasks were very simple. Only one subject, who had finished the 3rd grade, failed to give a correct answer to the following tasks: say the days of the week in indirect order, and point a geometrical (triangle) form in the comprehension exercises.

Results obtained in the memory exercises showed that, although very simple, not all stimuli can be recalled after a distraction. Thus, for a normal sample of subjects with elementary and secondary education, it is more difficult to recall than to recognize stimuli. As for recognition, the results were analyzed separately by stimuli type, and later grouped by material-specific both verbal and visual. Table 2 shows, for each memory exercise, the estimates that correspond to the rate of correct answers in the two models, separated by education degree.

The results describe above have shown that stimuli recall covers around $60 \%$ of the material presented, but stimuli recognition however, both of verbal and visual material, is above $80 \%$. Therefore, assessing memory through recognition is the most reliable parameter to analyze WT results.

Asymmetry between models - Comparing results from models $A$ and $B$, there are no statistically significant differences. The results obtained have shown that both models are the same and may be used for asymmetry analysis in the WT. Different performance of normal subjects in memory tests have shown that between the two models there may be a variation of up to 7 points.

Material-specific memory - The different types 
Table 2. Rate of correct answers in the two models, separated by education degree.

\begin{tabular}{lccccc}
\hline & \multicolumn{2}{c}{ Elementary education group } & & \multicolumn{2}{c}{ Secondary education group } \\
\cline { 2 - 3 } \cline { 5 - 6 } & Model A & Model B & & Model A & Model B \\
\hline Recall of objects & 0.85 & 0.83 & & 0.85 & 0.78 \\
Recall of words & 0.23 & 0.17 & & 0.28 & 0.23 \\
Recall of pictures & 0.60 & 0.64 & & 0.64 & 0.60 \\
Total recall & 0.56 & 0.55 & & 0.59 & $0.52 \mathrm{~A}$ \\
Recognition of objects & 1.0 & 1.0 & 1.0 & 1.0 \\
Recognition of words & 0.86 & 0.88 & & 0.88 & 0.86 \\
Recognition of abstracts designs & 0.85 & 0.79 & & 0.88 & 0.89 \\
Verbal recognition of pictures & 0.98 & 0.99 & & 0.99 & 1.0 \\
Visual recognition of pictures & 0.99 & 0.98 & & 1.0 & 0.98 \\
Recognition of verbal material & 0.95 & 0.96 & 0.96 & 0.96 \\
Recognition of visual material & 0.91 & 0.88 & 0.93 & 0.92 \\
\hline
\end{tabular}

Table 3. Comparing the correct answer, for the two models, on recognition of stimuli type.

\begin{tabular}{|c|c|c|c|c|c|c|}
\hline & & & \multicolumn{2}{|c|}{$\begin{array}{l}\text { Elementary } \\
\text { education group }\end{array}$} & \multicolumn{2}{|c|}{$\begin{array}{l}\text { Secondary } \\
\text { education group }\end{array}$} \\
\hline \multicolumn{3}{|c|}{ Stimulus type } & Model A & Model B & Model A & Model B \\
\hline Recognition of objects & $>$ & Recognition of words & 0 & 0 & $\mathrm{p}<0.0001$ & 0 \\
\hline Recognition of objects & $>$ & Recognition of abstracts designs & $\mathrm{p}<0.0001$ & $\mathrm{p}<0.0001$ & $\mathrm{p}<0.0001$ & $\mathrm{p}<0.0001$ \\
\hline Recognition of objects & $=$ & Verbal recognition of pictures & $p=0.0385$ & $\mathrm{p}=0.3219$ & $\mathrm{p}=0.3179$ & $\mathrm{p}=1$ \\
\hline Recognition of objects & $=$ & Visual recognition of pictures & $p=0.3219$ & $\mathrm{p}=0.0385$ & $\mathrm{p}=1$ & $p=0.074$ \\
\hline Recognition of words & $=$ & Recognition of abstracts designs & $\mathrm{p}=0.7984$ & $\mathrm{p}=0.0367$ & $\mathrm{p}=1$ & $p=0.2676$ \\
\hline Recognition of words & $<$ & Verbal recognition of pictures & $\mathrm{p}<0.0001$ & $\mathrm{p}<0.0001$ & $\mathrm{p}<0.0001$ & $\mathrm{p}<0.0001$ \\
\hline Recognition of words & $<$ & Visual recognition of pictures & $\mathrm{p}<0.0001$ & $\mathrm{p}<0.0001$ & $\mathrm{p}<0.0001$ & $\mathrm{p}<0.0001$ \\
\hline $\begin{array}{l}\text { Recognition of abstracts } \\
\text { designs }\end{array}$ & $<$ & Verbal recognition of pictures & $p<0.0001$ & $p<0.0001$ & $\mathrm{p}<0.0001$ & $\mathrm{p}<0.0001$ \\
\hline $\begin{array}{l}\text { Recognition of abstracts } \\
\text { designs }\end{array}$ & $<$ & Visual recognition of pictures & $p<0.0001$ & $\mathrm{p}<0.0001$ & $\mathrm{p}<0.0001$ & $p=0.00064$ \\
\hline $\begin{array}{l}\text { Verbal recognition of } \\
\text { picture }\end{array}$ & $=$ & Visual recognition of pictures & $p=0.1677$ & $p=0.1677$ & $p=0.3179$ & $\mathrm{p}=0.0748$ \\
\hline
\end{tabular}

of stimuli were compared two by two. Recalling objects is significantly easier than recalling words in both models; recalling objects is also easier than verbally recalling the pictures; verbally recalling the pictures is significantly easier than recalling words.

Recognizing objects or pictures is significantly easier than recognizing words and designs. Table 3 describes the results obtained for each comparison.

\section{DISCUSSION}

The accuracy of memory assessment in the WT depends on the similarity between the two models, because the results are interpreted by the rate of correct answers and the asymmetry between the performance of both the brain hemispheres. Comparing performance in the two stages of the test (anesthetizing each hemisphere separately) enables 
to analyze the asymmetry between the functional memory capacity in each hemisphere. From such comparison one draws conclusions about the functional memory reserve in the contralateral hemisphere (the one not proposed for surgery) and the likelihood of memory loss if the functional memory capacity of the hemisphere that will undergo surgery is still very preserved ${ }^{15,16}$. Therefore, the analysis of the more adequate types of stimuli fine tunes the exam and enhances the discussion on the models. In order to have a more reliable effect of anesthesia in one of the brain hemispheres, it is necessary that as many stimuli as possible can be recognized.

The main objective of this study was to help provide a more reliable WT protocol, better suited for the Brazilian population. Our results have shown that models $A$ and $B$ are equivalent, since the rate of correct answers was similar both in the elementary and secondary education groups. Subject performance in either of the two models was not significantly different. The chosen stimuli were simple enough for both the elementary and secondary education levels, and those data can be extrapolated for the higher education population.

Correct answer rates were analyzed for language and memory tasks. Language tasks presented no difficulty, since they were simple and easy to understand, as required by the test. Memory task results, on the contrary, were relevant.

A subject, in favorable testing conditions, is capable of recalling between $50 \%$ and $64 \%$ of the information that can be verbally decoded after a brief interference of attention tests. Such data have shown that results based exclusively on recall are not enough to determine if functional memory remains present. Therefore, recognition would be the most suitable parameter.

The criteria to consider a positive result when assessing memory in our study (which follows the Montreal procedures) vary between $60 \%$ and $68 \%$ of correct recognition answers. Normal subjects' results in our study are above $80 \%$ of correct recognition answers for all kinds of stimuli except abstract designs. Thus, we concluded that the analyzed models can provide data on functional reserve and functional adaptation of memory, since they are simple enough for a normal subject, under no medication, to recognize at least $80 \%$ of the stimuli.

Double encoding stimuli (objects and pictures) are significantly superior for memory assessment in the WT. Our results suggest that double encoding stimuli, because they are overall easier, ensure better test results and therefore it is more reliable to use this kind of material to make a post-surgery memory prognosis. On the other hand, material classified as unilateral in terms of encoding may be useful to assess memory-specific material, but it should be analyzed separately, taking the total correct answer rate into account, rather than asymmetry.

Christianson et al. ${ }^{17}$ noticed that words and pictures of common objects provided more conclusive results when compared to designs and faces, and stimuli diversity has the advantage of enabling a more detailed assessment of material-specific memory ${ }^{18}$.

The WT aims at studying how memory and language function in candidates for refractory epilepsy surgery. However, since it is a very short test, it is not able to encompass all complex aspects of those functions. Language cannot be fully assessed by having the patient repeat the days of the week or read isolated words only, nor can memory be assessed just by asking the patient to recognize four objects after a few-minutes interval. Therefore, the WT's structure enables no more than the assessment of episodic declarative memory within a very specific situation.

The study has shown that stimuli quality affects the results. Double encoding stimuli (pictures and objects that are easy to name), that is, theoretically encodable by both hemispheres, have shown higher correct answer rates. Stimuli classified as visual or verbal (unilateral in terms of encoding) are not memorized at the same rate. Therefore, the practical application of the observed parameters may also contribute to the study of declarative memory and material-specific memory functions.

Lastly, this study provides grounds for further discussion about the actual ability to anticipate memory deficits in patients taking the Test and submitted to temporal lobectomy.

\section{REFERENCES}

1. Cockerel O, Sander, JWA. O Custo econômico da epilepsia. In Costa JC Palmini A, Yacubian EMT, Cavalheiro E (eds). Fundamentos neurobiológicos das epilepsias: aspectos clínicos e cirúrgicos. São Paulo: Lemos, 1998;1;21-30.

2. Jones-Gotman M. Localization of lesion by neuropsychological testing Epilepsia 1991;32:Supp.5:41-52.

3. Hermann BP, Seidenberg M, Schoenfeld J, Davies K. Neuropsychological characteristics of the syndrome of medial temporal lobe epilepsy. Arch Neurol 1997;54:369-376.

4. Wada J. Clinical experimental observations of carotid artery injections of sodium amytal. Brain Cog, 1997;33:11-13. [Yale University Translation Comissioned by R.A. Novelly From J. Wada Igaku To Seibutsuqaku, $1949 ; 14: 221-222$ 
5. Milner B, Branch C, Rasmussen T. Study of short term memory after intracarotid injection of sodium amytal. Transactions of the American Neurological Association 1962;224-226.

6. Loring D, Meador K, Lee G, King D. Amobarbital effects and lateralized brain function - the Wada test, New York; Springer-Verlag 1992;1-23.

7. De Paola L. Epilepsia clinicamente refratária do lobo temporal associada a esclerose medial temporal. Dissertação (Mestrado), da Universidade Federal do Paraná Curitiba, 1999.

8. Dodrill CB. Preoperative criteria for identifying eloquent brain intracarotid amytal for language testing. Neurosurg Clin N Am,1993;4:2-4.

9. Rausch R, Silfvenius H, Wieser H, Dodrill CD, Meador KJ, Jones-Gotman M. Intraarterial amobarbital procedures. In Engel J. (ed). Surgical treatment of the epilepsies. New York: Raven Press, 1993:341-357.

10. Mader M J, Romano B W. The Wada test: diferences in Brazilian protocols. J Ep Clin Neurophysiol 2001:7;70-75

11. Risse G L, Gates J R, Fangman MC. A reconsideration of bilateral language representation based on the intracarotid amobarbital procedure. Brain Cog 1997;33:118-132.
12. Bertolucci P, Brucki S, Camapaci S, Juliano Y. O mini-exame do estado mental em população geral. Arq Neuropsiquiatr 1994;52:1-7.

13. Lezak M D. Neuropsychological assessment. 3.Ed. New York: Oxford Univ Press, 1995:373-669.

14. Spreen O, Strauss E. A compendium of neuropsychological tests: administration, norms and commentary New York: Oxford Univ Press, 1998:206-547.

15. Chelune G. Hipocampal adequacy versus functional reserve: predicting memory functions following temporal lobectomy. Arch Clin Neuropsychol 1995:10;413-432.

16. Trenerry MR, Loring, DW, Peerson RC, Scharbrough S. The Wada test. In Wyllie E. (ed). The treatment of epilepsy: principles and practice, 2.Ed. Baltimore: Williams \& Wilkins, 1996:1000-1005.

17. Christianson SA, Säisa J, Silfvenius H, Hemisphere memory differences in sodium amytal testing of epileptic patients. J Clin Exp Neuropsychol 1990;12:681-694.

18. Perrine K, Gershengorn J, Brown E R, Choi IS, Luciano DJ, Devinski O. Material-specific memory in the intracarotid amobarbital procedure. Neurology 1993;43:706-711. 